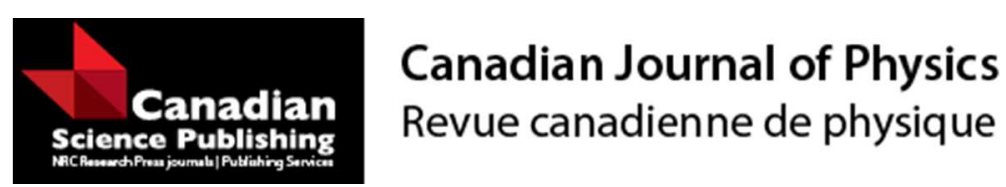

\title{
Photodetachment spectrum of hydrogen negative ion near a spherical surface
}

\begin{tabular}{|r|l|}
\hline Journal: & Canadian Journal of Physics \\
\hline Manuscript ID & cjp-2015-0583.R1 \\
\hline Manuscript Type: & Article \\
\hline Date Submitted by the Author: & 03-Nov-2015 \\
\hline Complete List of Authors: & $\begin{array}{l}\text { Ahmad, Afaq; Centre of Excellence in Solid State Physics } \\
\text { Iqbal, Azmat; Department of Physics, The University of Lahore } \\
\text { Ahmad, Iftikhar; Department of Physics, University of Malakand }\end{array}$ \\
\hline Keyword: & $\begin{array}{l}\text { quantum interference, photodetachment process, spherical surface, closed } \\
\text { orbit theory, Hydrogen negative ion }\end{array}$ \\
\hline &
\end{tabular}




\title{
Photodetachment spectrum of hydrogen negative ion near a spherical surface
}

\author{
A Afaq ${ }^{1 *}$, A Iqbal ${ }^{2}$ and A Iftikhar ${ }^{3}$ \\ ${ }^{1}$ Centre of Excellence in Solid State Physics, University of the Punjab, Quid-e-Azam Campus, Lahore-54590, \\ Pakistan \\ ${ }^{2}$ Department of Physics, The University of Lahore, Lahore-54590, Pakistan \\ ${ }^{3}$ Department of Physics, University of Malakand Chakdara, Dir (L), Pakistan \\ *Corresponding author. E-mail: aafaq.cssp@pu.edu.pk
}

\begin{abstract}
The semi-classical closed orbit theory is applied to study photodetachment of $\mathrm{H}^{-}$near an elastic spherical surface for a z-polarized laser light. It is assumed that similar to the outgoing detached-electron waves from the source, waves propagate from an image of the source behind the surface. We then calculate the classical action for those trajectories which are perpendicular to the surface. The spherical effects in total photodetachment cross section are controlled by curvature $\kappa$ of the surface. For zero curvature, our results match with the plane wall case while for a large curvature the results become asymptotic value of the cross section recently published.
\end{abstract}

Key Words: quantum interference; photodetachment process; spherical surface; closed orbit theory; hydrogen negative ion

PACS number(s): $32.80 \mathrm{Gc}, 03.65 \mathrm{Sq}$, 68.65.Fg, 32.60.+i

\section{Introduction}

The matter-surface interactions have much practical interest in the fundamental scientific research and technology; these interactions can provide information about adsorbate dynamics and surface properties which have many applications in material science, nanotechnology and microelectronics Most of the diagnostic techniques such as low energy electron diffraction (LEED), low energy ion scattering spectroscopy (LEISS), Auger electron spectroscopy (AES) are based on the scattering of atoms, ions, 
electrons, or photons from surfaces $[1,2]$. It is well known fact that the presence of a surface changes the dynamical properties of atoms, molecules or ions such as in photodetachment processes $[3,4,5]$.

The photodetachment of negative ions in external fields and/or surfaces has attracted significant attention after the development of semi-classical closed orbit theory (COT) put forth by Du and Delos [6, 7] to explain the oscillatory structure of photoabsorption cross section of atoms in magnetic fields. The COT beautifully demonstrates that a photodetached electron firstly propagates away from the source by absorbing photon energy and then it is reflected back to the source by external fields or surfaces, thus following a closed orbit. Each such classical closed orbit leads to oscillations in the photodetachment cross section. While in the absence of an external field or a surface, the photodetached electron waves never return to the nucleus and photodetachment cross section consists only smooth term. Thus, the COT nicely explains the physical scenario of oscillations in the photodetachment cross section by separating it into the smooth background and the oscillatory terms. The predictions of COT have also been verified by the photodetachmet microscopy experiments of negative ions $[8,9]$. Results of COT have been compared and verified by quantum mechanical results for some physical systems [10-14] that highlights the importance of this semi-classical theory. Theoretical imaging method (TIM) [15] is another useful technique to investigate the problems of photodetachment of negative ions near surfaces whose asymptotic results become identical to the COT results. The TIM emphasizes that the image is formed behind the surface when the source comes close to it and the reflected wave appears to come from the image.

The negatively charged hydrogen ions $\left({ }^{1} \mathrm{H}^{-}\right.$and $\left.{ }^{2} \mathrm{H}^{-}\right)$are of much interest in Stellar Astrophysics as they are the main source of continuum opacity in the photospheres of many stars [16-18]. The main contribution to the light absorption by $\mathrm{H}^{-}$comes from its photodetachment process:

$$
\mathrm{H}^{-}+h v=H+e^{-}
$$

The photodetachment of $\mathrm{H}^{-}$near an interface has attracted considerable interest since it has been proposed as a probe so as to investigate the adsorbate lifetime and charge transfer mechanism in backscattering process [19]. Firstly, Yang et al [20] investigated the photodetachment cross section of $\mathrm{H}^{-}$ near elastic wall by COT. Their formula is identical to that for electric field case obtained earlier and the wall-induced oscillations in the cross section were quite similar to those induced by static electric field 
$[21,22]$. Subsequently, Afaq and $\mathrm{Du}[15]$ re-investigated the photodetachment of $\mathrm{H}^{-}$near plane reflecting walls by introducing a new technique called theoretical imaging method and found walls-induced oscillations in the cross section similar to that of Yang et al [20].

Afterword, Yang et al [23] investigated photodetachment of $\mathrm{H}^{-}$near inelastic interface similarly but by defining a reflection coefficient $\alpha<1$; and they obtained different results from that of Ref. [20]. For $\alpha=0$, their results match with those of Zhao and $\mathrm{Du}$ [24] for the photodetachment of $\mathrm{H}^{-}$near a real metal surface. Afaq and Iftikhar [25] have revisited the same problem using COT by introducing a new parameter called reflection parameter which describes the inelasticity of the surfaces. Very recently, the authors $[26,27]$ have studied the most general cases for the photodetachment of $\mathrm{H}^{-}$near elastic and inelastic plane walls using COT.

Most of the previous research on the photodetachment of $\mathrm{H}^{-}$was focused near the plane surfaces or interfaces by COT, while a very few research work is recently presented near spherical surfaces by using TIM $[28,29]$. The objective of this paper is to study the photodetachment spectrum of $\mathrm{H}^{-}$near a hard spherical surface by COT and then compare the results with recently published work. In order to introduce the spherical effects in closed orbit theory, the classical action has been found as follows: after the photodetachment process, the detached-electron wave from the source approaches to the spherical surface at normal incident, simultaneously a wave appears to come from the image behind the surface. The total action then would be the sum of actions from source to the surface and the image to the surface. The model presented in this article for the physical system is very easy to understand the photodetachment of negative ions and it uses the COT near spherical surfaces first time in the literature. We hope that it may provide a help in photodetachment microscopy experiments near spherical surfaces.

The rest of the paper is organized as follows. In Section 2, we discuss the classical motion of the detached-electron from $\mathrm{H}^{-}$near a spherical surface and then obtain the analytical formula of total photodetachment cross section of this system based on closed orbit theory. In Section 3, we present key numerical results and compare them with the published results. In Section 4, we present some of the conclusions of the present work. 


\section{The total photodetachment cross section}

To calculate the total photodetachment cross section of hydrogen negative ion $\mathrm{H}^{-}$in the vicinity of an elastic spherical surface, we place $\mathrm{H}^{-}$at the origin while an elastic spherical surface is placed perpendicular to the z-axis at a distance $d$ atomic units from the source. When z-polarized laser light irradiates hydrogen negative ion near a spherical surface, the electrons of the ion may absorb a photon and excited to higher energy state. The energy of the detached-electrons may be sharply defined, but the initial direction of their motion is not specified. Thus detached-electrons may move in all possible directions. Some of the outgoing detached-electrons travelling along the classical trajectories never leave the hydrogen atom. They go out from and then return to the atomic nucleus after reflecting from the surface, thus forming closed orbits. These closed orbits satisfy the Bohr-Sommerfeld quantization condition and are related with the quantum interferences in COT.

To calculate the classical action, we follow; the outgoing classical trajectory move towards the surface, simultaneously the trajectories appear to move from the image towards the surface. The classical action is then calculated for those two trajectories that incident normally at the surface from the source and the image. A schematic representation of our model is shown in Fig.1.

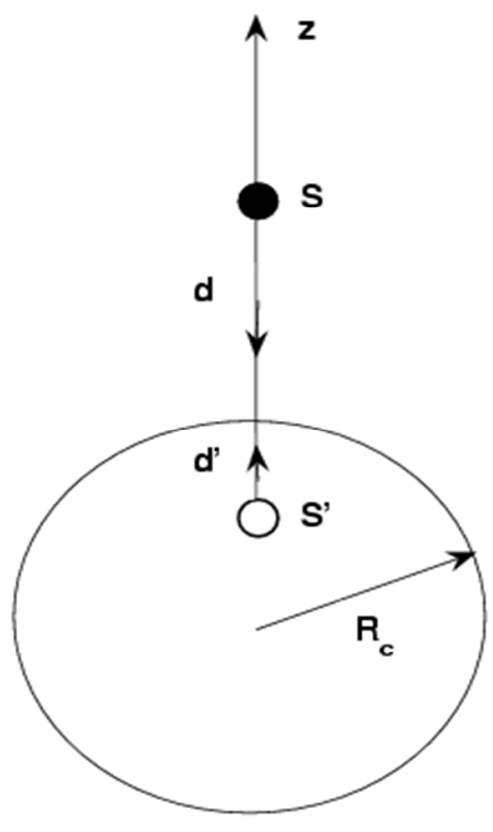


Figure 1. A schematic model representing the photodetachment of $\mathrm{H}^{-}$near an elastic spherical surface placed at a distance $d$ atomic units from the source. The detached-electron wave moves downward from the source $\mathbf{S}$ along the negative $\mathrm{z}$ direction while from the image $\mathbf{S}^{\prime}$, the detached-electron wave appears to move towards $\mathrm{z}$ direction and reaches the surface simultaneously.

To derive the expression for outgoing wave function, one has to solve the inhomogeneous Schrodinger equation given by $[21,22]$

$$
(E-H) \Psi^{+}(\mathbf{r})=D(\mathbf{r}) \Psi_{\mathrm{i}}(\mathbf{r})
$$

where $\Psi^{+}(\mathbf{r})$ is the outgoing wave function, $E$ is the K. E. of detached-electron, i.e., $E=E_{\mathrm{ph}}-E_{\mathrm{b}}$, where $E_{\mathrm{ph}}$ is photon energy and $E_{b}$ is binding energy of $\mathrm{H}^{-}, \Psi_{\mathrm{i}}$ is the initial wave function of $\mathrm{H}^{-}$and $D$ is the dipole operator $z=r \cos \theta$, for linear laser photons.

The short range spherically symmetric potential $V_{\mathrm{b}}(r)$, where $r$ is the distance of the active electron from the origin of the nucleus, binds the active electron of $\mathrm{H}^{-}$. The initial bounded wave function of $\mathrm{H}^{-}$is $\Psi_{\mathrm{i}}(\mathbf{r})=B e^{-k_{b} r} / r$, value of normalization constant $B$ is $0.31552, k_{\mathrm{b}}$ is the momentum of bound electron and can be written in terms of binding energy $E_{b}$ of $\mathrm{H}^{-}$as $k_{\mathrm{b}}=\sqrt{2 E_{\mathrm{b}}}$. The Hamiltonian $H$ governs the motion of the detached electron and is given by

$$
H=-\frac{1}{2} \nabla^{2}+V_{\mathrm{b}}(r)
$$

The potential $\mathrm{V}_{\mathrm{b}}(r)$ does not affect the motion of outgoing electron far from the nucleus and hence can be neglected. Physically valid solution of Eq. (1) that satisfies the correct outgoing boundary conditions must be an outgoing wave at a large $r$. The oscillator-strength density can be calculated from the detachedelectron wave function $\Psi^{+}$using the relation,

$$
D f(E)=-\frac{2 E_{p h}}{\pi} \mathrm{I}_{\mathrm{m}}\left\langle D(\mathbf{r}) \Psi_{\mathrm{i}}(\mathbf{r}) \mid \Psi^{+}(\mathbf{r})\right\rangle
$$

The photodetachment cross section is proportional to the oscillator-strength density $D f(E)$, and is given by 


$$
\sigma(E)=-\frac{4 E_{p h}}{c} \mathrm{I}_{\mathrm{m}}\left\langle D(\mathbf{r}) \Psi_{\mathrm{i}}(\mathbf{r}) \mid \Psi^{+}(\mathbf{r})\right\rangle
$$

where, $c$ is the speed of light.

In order to solve Eq. (1) near the nucleus, the photo-detached wave function $\Psi^{+}$is decomposed into two parts as is done in Refs. [21, 22]; one is a direct wave function and other is returning wave function such that $\Psi^{+}(\mathbf{r})=\Psi_{\text {dir }}(\mathbf{r})+\Psi_{\text {ret }}(\mathbf{r})$. Where $\Psi_{\text {dir }}(\mathbf{r})$ represents the steady outgoing detached-electron wave going out in the negative z-direction after photodetachment process and reaches to the surface. While $\Psi_{\text {ret }}(\mathbf{r})$ represents the returning wave appears to be coming from the image and it propagates in zdirection. These two waves superimpose near the nucleus, thus giving quantum interference. The wave function $\Psi_{\text {dir }}(\mathbf{r})$ is given by [21]

$$
\Psi_{\mathrm{dir}}(r, \theta, \phi)=-\frac{4 k^{2} B i}{\left(k_{b}^{2}+k^{2}\right)^{2}} \cos (\theta) h_{1}^{(1)}(k r)
$$

Where, $h_{1}^{(1)}(k r)$ is the outgoing Hankel function with its asymptotic approximation, $h_{1}^{(1)}(k r)=\frac{e^{i(k r-\pi)}}{k r}$, which is valid on the surface of sphere, while the outgoing wave on the surface is given by

$$
\Psi_{\mathrm{dir}}(R, \theta, \phi)=-\frac{4 k^{2} B i}{\left(k_{b}^{2}+k^{2}\right)^{2}} \cos (\theta) \frac{\exp (i k R)}{k R}
$$

As the wave propagates out from the surface of the sphere, both its phase and amplitude will change. Semiclassically, the wave propagating out of this sphere can be expanded in terms of outgoing wave in Eq. (6) as

$$
\Psi(q)=\sum_{j} A_{j}\left(\boldsymbol{q}_{0}\right) e^{i\left(s_{j}(\boldsymbol{q})-\frac{\mu_{j} \pi}{2}\right)} \Psi_{\mathrm{dir}}^{j}\left(\boldsymbol{q}_{0}\right)
$$

After reflection from the spherical surface, this wave is known as retuning wave. This returning wave appears to be come from the image of the source. The returning wave will then interact with the direct wave from the source and leads to oscillation in the photodetachment spectra of $\mathrm{H}^{-}$. The returning wave can be considered as a plane wave propagating along the closed orbit in the z-direction and is given by

$$
\Psi_{\text {ret }}(\mathbf{r})=M e^{i k z}
$$


The proportionally constant $M$ contains information about the outgoing detached-electron wave along $j$ th trajectory before it returns to the source and is given by

$$
M=\sum_{j} A_{j}\left(\boldsymbol{q}_{0}\right) e^{i\left(s_{j}(\boldsymbol{q})-\frac{\mu_{j} \pi}{2}\right)} \Psi_{\mathrm{dir}}^{\mathrm{j}}\left(\boldsymbol{q}_{0}\right)
$$

The summation includes all possible trajectories such that each trajectory begins at a point $\boldsymbol{q}_{\mathbf{0}}$ on the surface of the hypothetical sphere around nucleus and arrive at the point $\boldsymbol{q}$. The classical action $S_{j}(\boldsymbol{q})$ is for the $j$ th trajectory given by

$$
S_{j}(\boldsymbol{q})=\int_{q_{0}}^{q} \boldsymbol{P} \cdot \mathrm{d} \boldsymbol{q}
$$

$A_{j}\left(\boldsymbol{q}_{0}\right)$ is the classical amplitude,

$$
A_{j}\left(\boldsymbol{q}_{0}\right)=\left[J\left(t_{0}, \boldsymbol{q}_{0}\right) / J(t, \boldsymbol{q})\right]^{1 / 2}
$$

With, $J(t, \boldsymbol{q})=\rho(t)\left|\begin{array}{ll}\frac{\partial z}{\partial t} & \frac{\partial z}{\partial \theta} \\ \frac{\partial \rho}{\partial t} & \frac{\partial \rho}{\partial \theta}\end{array}\right|$ being a Jacobian, and $\mu_{j}$ is the Maslove index for jth trajectory.

In our particular case, the classical action in Eq. (10) would be $S_{j}=k L_{j}$ with $L_{j}=n d$, is the length of the jth trajectory. The dimensionless number $n=1+1 /(1+2 \kappa d)$. This action is calculated by sum of the actions of direct and image trajectories in our case that is, $S_{j}(\boldsymbol{q})=\int_{q_{0}}^{q} \boldsymbol{p} . d \boldsymbol{q}=\int_{0}^{d} k d z+\int_{0}^{d^{\prime}} k d z=$ $k\left(d+d^{\prime}\right)$, where $d^{\prime}=d /(1+2 \kappa d)$ is a mirror equation [28] with $\kappa$ is a curvature of the spherical surface. The curvature $\kappa$ is the property of the spherical surfaces deviates from being flat and it may vary from zero to infinity as extreme values. The classical amplitude $A_{j}$ in our case would be (for detailed derivation see Appendix),

$$
A_{j}=\frac{R}{R+n d}
$$

With the approximation of small $R$, Eq. (9) can be written as

$$
M=\left(\frac{4 k^{2} B i}{n d\left(\mathrm{k}_{\mathrm{b}}^{2}+\mathrm{k}^{2}\right)^{2}}\right) e^{i\left(s_{j}-\frac{\pi}{2}\right)}
$$

Using Eq. (13) into Eq. (8), the returning wave can be written as 


$$
\Psi_{\mathrm{ret}}(\mathbf{r})=\left(\frac{4 k^{2} B i}{n d\left(k_{b}^{2}+k^{2}\right)^{2}}\right) e^{i\left(S_{j}-\pi / 2\right)} e^{i k r \cos \theta}
$$

The overlap integral of the returning wave in Eq. (14) with the source provides the oscillating term in the oscillator-strength density:

$$
\begin{aligned}
D f_{1}(E, d, \kappa) & =-\frac{2 E_{p h}}{\pi} \mathrm{I}_{\mathrm{m}}\left\langle D \Psi_{\mathrm{i}} \mid \Psi_{\text {ret }}\right\rangle \\
& =-\mathrm{D} f_{\mathrm{o}}(\mathrm{E}) \frac{\sin \left(S_{j}-\pi / 2\right)}{S_{j}}
\end{aligned}
$$

The total oscillator-strength density in the presence of a spherical wall is the given by

$$
D f(E, d, \kappa)=D f_{0}(E)\left[1-3 \frac{\sin \left(s_{j} \frac{\pi}{2}\right)}{s_{j}}\right] \text {. }
$$

where $D f_{0}(E)=\frac{8 \sqrt{2} B^{2} E^{3 / 2}}{3\left(E_{b}+E\right)^{3}}$, is the smooth background term [22]. Thus, total photodetachment cross section of $\mathrm{H}^{-}$near an elastic spherical surface is given by

$$
\sigma(E, d, \kappa)=\sigma_{0}(\mathrm{E})\left[1+3 \frac{\cos s_{j}}{s_{j}}\right]
$$

where $\sigma_{0}(E)=16 \sqrt{2} \pi^{2} B^{2} E^{3 / 2} / 3 c\left(E+E_{b}\right)^{3}$, is the smooth background photodetachment cross section. Eq. (17) gives the total photodetachment cross section of $\mathrm{H}^{-}$near an elastic spherical surface which is the sum of the smooth background and oscillatory terms. The smooth background term is the total photodetachment cross section in the absence of a spherical surface. The oscillatory term results from the quantum interference of the steady outgoing detached-electron wave and the classical returning electron wave.

\section{Numerical results and discussion}

Fig. 2 represents the numerical results of the formula in Eq. (17) for different values of the curvature that defines spherical nature of the surface. The source-surface distance is fixed to 25 atomic units in these calculations. The solid red line is our result and the blue dashed lines are for plane wall case [20] for comparison. The total cross section is simply a superposition of a background term and a sinusoidal oscillatory term. Actually, this spectrum is almost exactly the same as the one given by the following 
previous known formula for the detachment cross section of $\mathrm{H}^{-}$near a plane wall case (zero curvature case) [20]

$$
\sigma(E, d, \kappa=0)=\sigma_{0}(\mathrm{E})\left[1+3 \frac{\cos (2 \sqrt{2 E} d)}{2 \sqrt{2 E} d}\right]
$$

Fig. 2(d) represents this result. The influence of spherical surface is presented in Fig. 2(a-c). It shows that by decreasing the value of curvature, the spherical wall problem indicated by the solid red line reduces to the plane wall case represented by dashed lines and it becomes exactly the same for zero curvature as is shown in Fig. 2(d). We also observed that our formula in Eq. (17) becomes the limiting value of the formula derived by theoretical imaging method [28].

The formula in Eq. (17) can also be derived by using quantum mechanical approach as for plane elastic wall case as adopted in Ref. [11], where the photodetacment cross section in terms of dipole matrix element is

$$
\left.\sigma(E)=\frac{4 \pi^{2} E_{p h}}{c}\left|{ }^{2} f_{E}\right| D \mid i\right\}^{2} \mathrm{~d} E
$$

Where $c$ is the speed of light, $E_{p h}$ is the photon energy, $D=\boldsymbol{r} . \hat{e}$ is the dipole operator which is equal to $z=r \cos \theta$ for photon polarized along $z$ direction, $\sigma_{E} \mid$ is the final-state wave function of the detached electron, the final state is normalized according to $\left\langle f_{E} \mid f_{E^{\prime}}\right\rangle=\delta\left(E-E^{\prime}\right)$. The $\mid i$ ? is the initial-state wave function of bound state of hydrogen negative ion $\mathrm{H}^{-}$. If we neglect the short range Coulomb potential of atomic core of $\mathrm{H}^{-}$, the detached electron moves freely while propagating away from the core before the infinite potential spherical wall placed at $-d$ from the source. Then normalized final state wavefunction in cylindrical coordinates can be obtained by solving the Schrodinger equation in free space with the boundary condition, $\psi(-d)=0$. Where $d$ is related to the curvature of the spherical surface according to the mirror equation as $d^{\prime}=d /(1+2 \kappa d)$, where $d^{\prime}$ is the distance of the image from spherical surface and $\kappa$ is the curvature of the spherical surface.

In order to calculate the dipole matrix element in Eq. (19), we need to transform the final state wave function of the detached electron from cylindrical coordinates to spherical coordinates using the frame 
transformation technique developed by Fano [30], Hermin [31] and Greene [32]. In this way, we will obtain the expression of photodetachment cross section using Eq. (19).

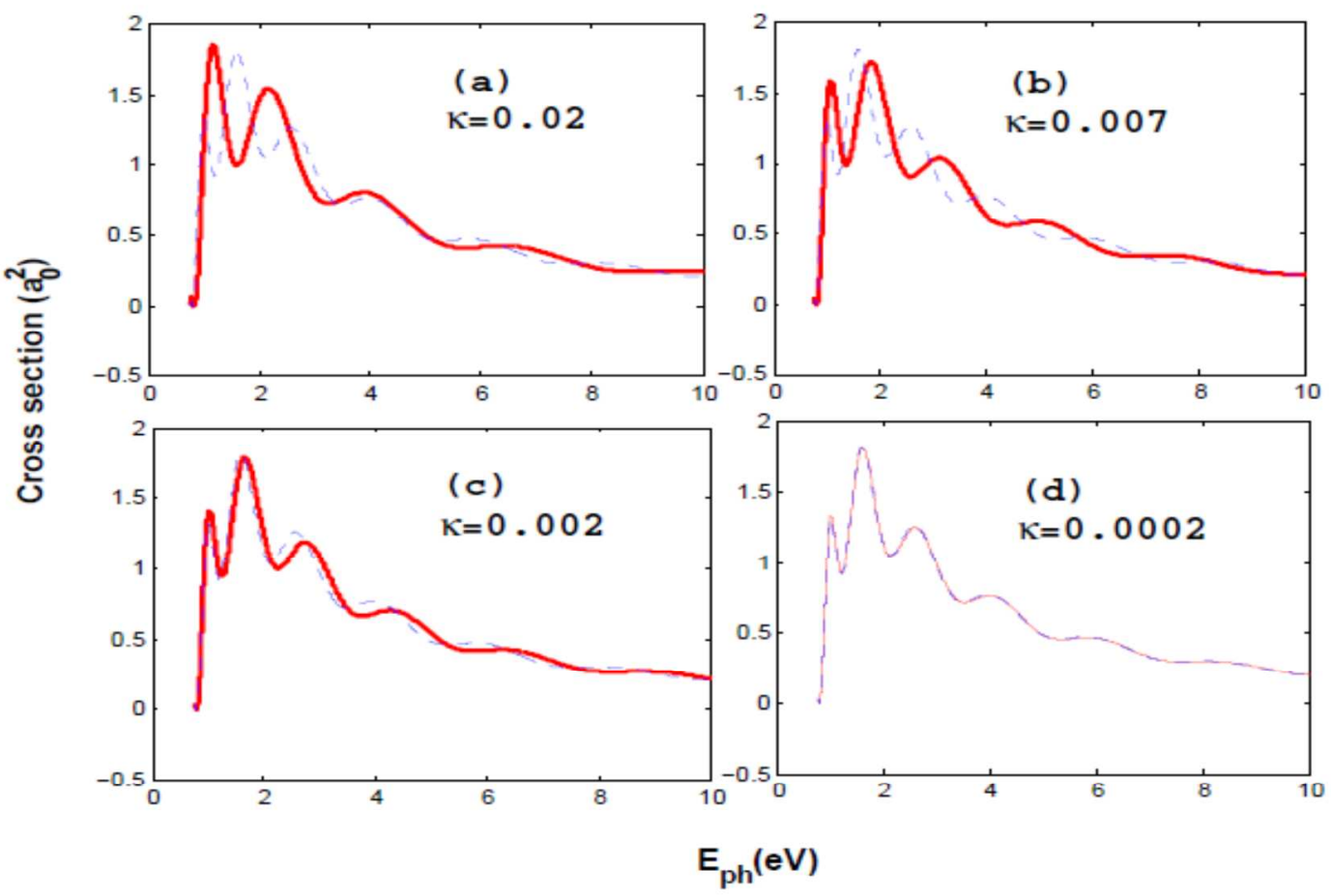

Figure. 2. Photodetachment spectra of $\mathrm{H}^{-}$near spherical surface. Solid red line is our result and the dashed blue lines are for plane wall case for comparison.

\section{Conclusion}

We derived an analytical formula for the photodetachment cross section of $\mathrm{H}^{-}$near a spherical surface using closed orbit theory. The resulting spectrum is an oscillatory structure which is very different from that for a plane wall case. In order to introduce spherical effects in the total cross section in terms of curvature $\kappa$, the classical action is calculated by imaging technique. The oscillations in the spectrum can be controlled by curvature $\kappa$. For zero curvature, our results coincide well with the results of plane wall case. We hope that our model may provide a help to experimentalists in order to study the ion trap in spherical surface. 


\section{Appendix}

In the cylindrical coordinates the outgoing detached electron wave dynamics may be explained by:

$$
\begin{gathered}
\rho(t)=R \sin \theta+k t \sin \theta \\
z(t)=R \cos \theta+\boldsymbol{k} t \cos \theta
\end{gathered}
$$

Where, $R$ is the radius of the hypothetical sphere in the closed orbit theory. Using the definition of Jacobian in Eq. (11) and Eq. (A. 1), one can find,

$$
\begin{aligned}
J(t, \boldsymbol{q}) & =\rho(t)\left|\begin{array}{ll}
\frac{\partial z}{\partial t} & \frac{\partial z}{\partial \theta} \\
\frac{\partial \rho}{\partial t} & \frac{\partial \rho}{\partial \theta}
\end{array}\right| \\
& =k(R+k t)^{2} \sin \theta \\
\Rightarrow J\left(t_{0}, \boldsymbol{q}\right) & =\left|k(R+k t)^{2} \sin \theta\right|_{t=0}=k R^{2} \sin \theta
\end{aligned}
$$

The classical amplitude in Eq. (11) can be obtained as:

$$
A_{j}=\frac{R}{R+\boldsymbol{k} t}
$$

Where in our case of spherical surface, $\boldsymbol{k} t=d+d^{\prime}=d+\frac{d}{1+2 \kappa d}=n d$. Therefore the classical amplitude as in Eq. (12),

$$
A_{j}=\frac{R}{R+n d}
$$




\section{References}

[1] Lin C D Review of Fundamental Process and Applications of atoms and lons (World Scientific, London, 1993) p 517.

[2] Mahanty J and Ninham B W Dispersion Forces (Academic, London, 1976).

[3] De-hua Wang , Hong-run Wang and Tian-qi Liu, Dong-qi Liang 2010 Chin. J. Phys. 48767.

[4] Rous P R and Hartley D M 1995 Chem. Phys. 236299.

[5] Hartely D M and Raous P J 1995 Surf. Sci. 341213.

[6] Du M L and Delos J B 1987 Phys. Rev. Lett. 581731.

[7] Du M L and Delos J B 1988 Phys. Rev. A 38 1896, Du M L and Delos J B 1988 Phys. Rev. A 381913.

[8] Blondel C, Delsart C and Dulieu F 1996 Phys. Rev. Lett. 773755.

[9] Bracher C, Kramer T and Kleber M 2003 Phys. Rev. A 67043601

[10] Guangcan Yang, Yizhuang Zheng and Xianxi Chi 2006 Phys. Rev A 73043413.

[11] Guang-can Yang, Kui-kui Rui and Yi-zhuang Zheng 2007 Chin. J. Chem. Phys. 20537.

[12] Zhao H J and Du M L 2007, Phys. Rev. A 76017401.

[13] Wu Xiao-Qing, Du Meng-Li and Zhao Hai-Jun 2012 Chin. Phys. B 21043202.

[14] Zhao H J, Ma Z J and Du M L 2015, Physica B 46654.

[15] Afaq A and Du M L 2007 J. Phys. B 401309.

[16] Chandrasekhar S 1945 Astrophys. Journal 102223.

[17] Ohmura T and Ohmura H 1960 Phys. Rev. 118154.

[18] Frolov A M and Smith V H Jr. 2003 J. Chem. Phys. 361739.

[19] Sjakste J, Borisov A G and Gauyacq J P 2004 Phys. Rev. Lett. 92156101

[20] Guangcan Yang, Yizhuang Zheng and Xianxin Chi 2006 J. Phys. B 391855. 
[21] Du M L 2004 Phys. Rev. A 70055402.

[22] Du M L 2006 Eur. Phys. J. D 38533.

[23 ] Yang G G, Zheng Y and Chi X 2007 J. Theo. Comp. Chem. 6353.

[24] Zhao H J and Du M L 2009 Phys. Rev. A 79023408.

[25] Afaq A and Iftikhar Ahmed 2011 Int. J. Quantum Chem. 1114067.

[26] Azmat lqbal and Afaq A 2015 Chin. Phys. B 24, 083201.

[27] Azmat lqbal, Afaq A and Raja Amjad J 2015 Int. J. Quantum Chem. DOI: 10.1002/qua.24965

[28] Haneef M, Iftikhar Ahmad, Afaq A and Rahman A 2011 J. Phys. B. 44195004.

[29] Haneef M, Afaq A, Iftikhar Ahmed and Rahman A 2012 Chin. Phys. Lett. 29013202.

[30] Fano U 1981 Phys. Rev. A 24619.

[31] Harmin D A 1982 Phys. Rev. A 262656.

[32] Green C H 1987 Phys. Rev. A 364236. 